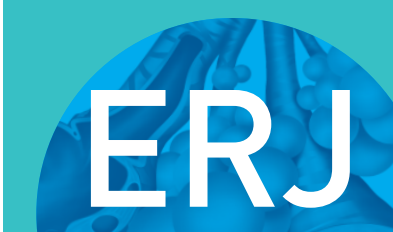

open research
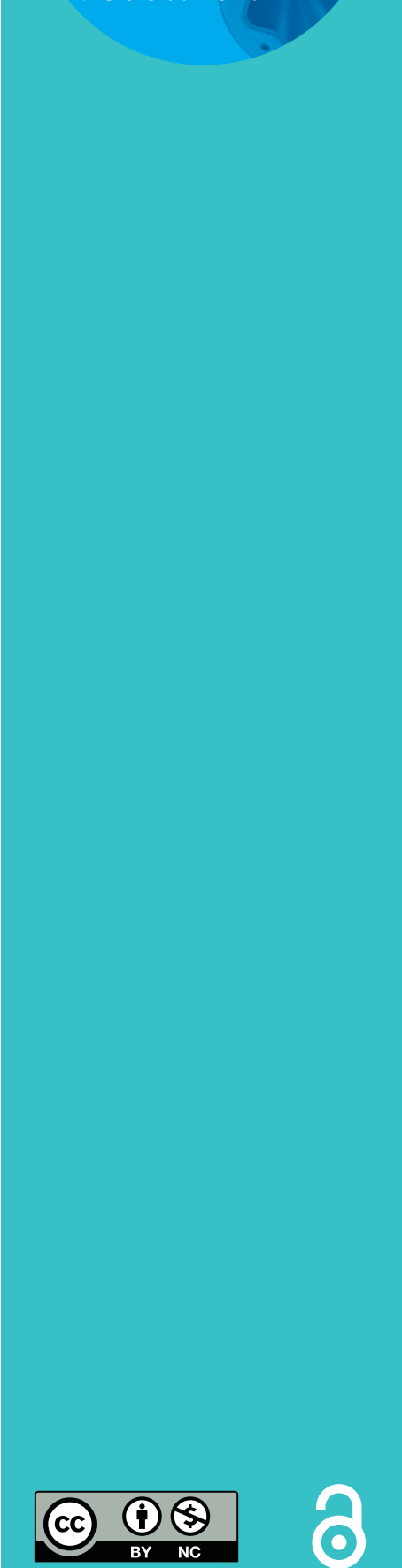

\section{Highlights of thoracic oncology from the 2018 ERS International Congress}

\author{
Polyxeni Ntontsi ${ }^{1}$, Clementine Bostantzoglou ${ }^{2}$ and Torsten Gerriet Blum ${ }^{3}$
}

Affiliations: ${ }^{1} 7$ th Respiratory Medicine Dept, "Sotiria" Athens Chest Diseases Hospital, Athens, Greece. ${ }^{2}$ Intensive Care Unit, Korgialeneion-Benakeion General Hospital, Athens, Greece. ${ }^{3}$ Lungenklinik Heckeshorn, Helios Klinikum Emil von Behring, Berlin, Germany.

Correspondence: Polyxeni Ntontsi, 7th Respiratory Medicine Dept, “Sotiria” Athens Chest Diseases Hospital, Athens, Greece. E-mail address: xenia-1990ahotmail.com

ABSTRACT Lung cancer is a substantial global burden for patients, healthcare professionals and healthcare systems. Multiple scientific international and national initiatives are tackling the various problems associated with this disease, which is currently the leading cause of cancer-related mortality worldwide. During the European Respiratory Society International Congress 2018 in Paris, France, lung cancer experts gathered to present the most recent aspects of lung cancer care, and discuss the need for joint initiatives and an international lung cancer alliance, aiming to provide high quality, accessible health care. The US experience and American Lung Association/American Thoracic Society implementation guide on lung cancer screening programmes, the key features of optimising and implementing such programmes, the challenges of treatment in the subset of patients where lung cancer is combined with interstitial lung disease, and novel lung cancer biomarkers and immunotherapy were among the most anticipated issues covered during the congress.

@ERSpublications

Lung cancer is a serious global burden for patients and healthcare systems. Experts from multiple scientific societies gathered during \#ERSCongress 2018, to present the most recent aspects on care and stress the need for joint initiatives. http://bit.ly/2VK2S4P

Cite this article as: Ntontsi P, Bostantzoglou C, Blum TGHighlights of thoracic oncology from the 2018 ERS International Congress. ERJ Open Res 2019; 5: 00222-2018 [https://doi.org/10.1183/ 23120541.00222-2018].

Copyright $\odot$ ERS 2019. This article is open access and distributed under the terms of the Creative Commons Attribution Non-Commercial Licence 4.0. 
Lung cancer is the number one cause of cancer-related mortality worldwide. According to the World Health Organization, it will account for $>1.76$ million deaths in 2018, whilst it will affect almost 2 million people [1]. These devastating numbers constitute a substantial global burden not only for lung cancer patients but also for healthcare professionals and systems. Multiple international and national scientific initiatives are tackling the various problems associated with this debilitating and deadly disease. In a well-attended session during European Respiratory Society (ERS) International Congress 2018 in Paris, France, lung cancer experts representing the American Lung Association (ALA)/American Thoracic Society (ATS), European Society for Medical Oncology (ESMO), Japanese Respiratory Society and ERS gathered to highlight the most recent aspects of care from their individual perspectives, underlining the need for an international lung cancer alliance.

Considering that the stage of the disease at diagnosis directly correlates with survival rates and treatment options, and that unfortunately, the majority of new diagnoses are made at an advanced stage of the disease, when treatment with curative intent is not applicable, lung cancer is an excellent candidate for the development of screening programmes, aiming at earlier diagnosis in order to improve cure rates and reduce lung cancer mortality.

Carey Thomson, Chief of Pulmonary and Critical Care Medicine, and Associate Chair of the Dept of Medicine at Mount Auburn Hospital in Cambridge, MA, USA, and Associate Professor at Harvard Medical School, Cambridge, reported on the US experience in lung cancer screening (LCS). Dr Thomson's field of expertise and current work within the ATS and the ALA covers implementation of LCS programmes. She reviewed the current knowledge on LCS and LCS guidelines in the USA, analysed the barriers to implementation and strategies applied in the USA to overcome them, and finally presented the ALA/ATS implementation guide on LCS.

Lung cancer numbers are still devastating in the USA with an estimated 200000 new diagnoses and 160 000 deaths in 2018 [2]. In 1992, the Early Lung Cancer Action Program (ELCAP) assessed the benefit of annual computed tomography (CT) screening for lung cancer. It showed a high proportion of patients diagnosed at stage 1 and triggered more research on LCS [3]. The National Lung Screening Trial (NLST) trial ran from 2002 to 2010 and enrolled 53454 individuals fulfilling the following inclusion criteria: age $55-74$ years; smoking history of $\geqslant 30$ pack-years; current or former smokers who had quit $<15$ years before inclusion without symptoms of lung cancer and with a potential benefit from treatment. NLST was the first large prospective multicentre trial that managed to show a lung cancer mortality reduction of $20 \%$ in individuals who were randomised to the LCS arm, compared to those in the control arm, with conventional chest radiography screening. Furthermore, an all-cause mortality reduction of $6 \%$ was shown in the LCS arm of the study. However, apart from this excellent result, NLST raised the question of who would benefit from such a screening programme given the fact that within the trial, benefit from LCS varied depending on lung cancer risk among screened individuals. However, the potential harms arising from a LCS programme came to light, such as physical complications from unnecessary invasive procedure as well as risk of future cancer from radiation exposure [4].

Current recommendations of the US Preventive Services Task Force include LCS programmes based on extended NLST criteria (age 55-80 years) [5]. The National Comprehensive Cancer Network (NCCN) distinguished two groups of high-risk factors in their LCS recommendations: group 1 with patients 55-74 years old according to the NLST criteria, and group 2 with patients $\geqslant 50$ years old with a smoking history of $\geqslant 20$ pack-years and no limit on quit history, plus an additional risk including history of lung cancer on first degree, occupational exposures, residential radon, chronic lung disease (idiopathic pulmonary fibrosis (IPF) or chronic obstructive pulmonary disease) or a personal history of smoking-related cancer [6]. For individuals in the first group, NCCN makes a category 1 recommendation (based on high-level evidence and broad consensus among panel members) for annual low-dose computed tomography (LDCT) screening. For those in the second group, LDCT screening is a category $2 \mathrm{~A}$ recommendation (lower-level evidence and broad consensus among panel members).

There is a number of important issues to be addressed when aiming to achieve high quality of screening. First, attention needs to be brought to patient selection and eligibility criteria. Another key element is the implementation of standardised protocols to perform, interpret and report CTs, and to guide lung nodule evaluation. There is an added value when LSC is run by multidisciplinary teams (MDTs). Furthermore, there is a need for patient and provider educationin terms of shared decision making, organised registries of all screened patients and tracking of nodule evaluation as well as a need for smoking cessation interventions integrated into LCS programmes [7]. These approaches aim to balance the benefit and harm of such a programme, and address overdiagnosis.

In 2015, Medicare and Medicaid services approved LCS in the USA for individuals 55-77 years old, current or former smokers with $\geqslant 30$ pack-years who quit $\leqslant 15$ years before initiation, with no clinical 
condition that would limit life expectancy and no signs or symptoms of lung cancer. Individuals from NCCN group 2 were not added. Mandates were offered for a face-to-face visit during which high risk was confirmed and clinical decisions were made, as well as a smoking cessation counselling. Centres required a designation by the American College of Radiology (ACR) and data submission to the ACR registry. Despite insurance services offering LCS, according to ATS data, in 2015, only $3.9 \%$ of the 6.8 million patients eligible for LCS were screened, with no expectation of improvement for the current year. Lack of knowledge among smokers, lack of physician awareness and lack of access to LDCT screening are among the reasons believed to be accountable for these low participation numbers.

The ALA/ATS implementation guide on LCS was developed as a guide and web-based toolkit to address common challenges associated with implementing a LCS programme across diverse settings [8]. Leaders in LCS from numerous institutions worked on this project incorporating the presence of International ELCAP, national lung cancer roundtables and statewide programmes, as well as multidisciplinary expertise. The programme aimed to serve as a survey of current practice and operational strategies rather than a guideline or position statement, focusing on practical, operational tips.

The structure of each LCS programme depends on available resources, and the type of institution and practice, but also on the skills and interest of individual providers. There are two core types of LCS programme, centralised and decentralised. A centralised LCS programme actively recruits eligible patients, conducts shared decision-making visits, assists in smoking cessation, performs and interprets all screening studies, arranges consultations and follow-up studies, tracks all outcome data, and finally communicates results and follow-up plans to patients and referring providers. This model requires significant resources including a dedicated LCS coordinator, clinical leadership and an integrated MDT programme. Conversely, a decentralised LCS programme performs examinations and leaves their interpretations and all further functions to the referring provider. In between, there are hybrid LCS programmes, which equally share features of centralised and decentralised processes. Overall, planning of LCS programmes requires engagement of local leadership, MDTs and trained radiology working groups, as well as appropriate insurance policies, and adequate outreach to providers and eligible participants.

David R. Baldwin, Consultant Respiratory Physician at Nottingham University Hospitals and Honorary Professor at the School of Medicine, University of Nottingham, Nottingham, UK, presented the key issues in optimising and implementing LDCT LCSs. These include proper selection of individuals at high risk and their enrolment in these programmes, optimisation of the scanning process, management of indeterminate and incidental findings, introduction of optimal clinical work-up and subsequent treatment, and consideration of cost effectiveness.

Although LCS programmes are covered in North America, Europe so far lacks organised nationwide screening programmes. The European Society of Radiology/ERS published a white paper on LCS in 2015 and likewise, the European Society of Thoracic Surgeons, its recommendations in 2017; however, a European standard for LDCT screening is still eagerly expected.

The UK Lung Cancer Screening (UKLS) study was a randomised controlled trial of LDCT screening that recruited high-risk individuals, aged 50-75 years, using a population approach. Data were collected from 4061 individuals who consented to participate and 2756 who declined participation; of those declining participation, $27.1 \%$ completed a nonparticipation questionnaire. Based on the experience from the UKLS study, factors associated with reduced trial enrolment included female sex, older age, current smoking, lower socioeconomic group and higher affective risk perception [9]. Another issue to be addressed is proper selection of patients who are at high risk for lung cancer. Multivariable population selection based on elaborated risk prediction models has shown better results rather than selection based on age and smoking only. Yet, more research is needed to determine the variants to be included in an optimum risk prediction model [10].

Regarding radiological technical standards, specific CT protocols are needed in order to ensure diagnostic quality while maintaining the lowest possible radiation doses. A volumetric approach to nodule detection and measurement is considered superior to two-dimensional measurements, and is now incorporated into European studies [11, 12]. Novel image reconstruction techniques are expected to increase nodule detection rates using even lower radiation doses. Nodule assessment is also crucial for the success of a screening programme. In order to address this issue and to standardise interpretation, reporting and management of nodules in LDCT screening, the ACR has established the Lung-RADS classification (Lung CT Screening Reporting and Data System), which needs, however, to be prospectively validated [13].

Another open issue is the optimal time interval between screening rounds. Biennial screening has been proven to be as effective as annual, with the obvious additional benefit of better cost effectiveness [14]. 
Management of indeterminate findings is another important aspect of LCS programmes. Strategies to minimise unnecessary work-up and reduce potential physical harms associated with LCS include specific guideline-driven protocols for nodule categorisation and volumetry to better assess growth. Optimal work-up is also a key feature of a successful LCS programme. Such a work-up should include better prediction of malignancy, improved and more accurate localisation of nodules, and minimally invasive and less traumatic surgical approaches.

An additional benefit of LCS is the reliable detection of certain comorbidities that are frequent among smokers, including coronary artery calcification, emphysema, interstitial lung disease (ILD) and osteoporosis. Moreover, incorporation of smoking cessation programmes into LCS is linked to increased cessation rates; smoking cessation rates in UKLS were higher than UK population cessation rates in all groups, whether the individual had received an intervention or not.

Finally, LCS should allow adequate information and support of participants, shared decision planning, and be based on country- and system-specific resources and multidisciplinary approaches.

Multiple studies suggest a link between ILD and lung cancer through various pathogenetic mechanisms, including inflammation, resistance to apoptosis and extracellular matrix accumulation [15]. Epidemiological studies demonstrate a high risk for IPF patients to develop lung cancer. Among IPF patients, male smokers seem to be at higher risk for lung cancer and the most common histology appears to be squamous [16].

Yoichi Nakanishi, Professor in the Dept of Respiratory Medicine at the Kyushu University of Japan, Fukuoka, Japan, and president of the NPO-West Japan Oncology Group, is leading, among others, clinical trials on novel therapeutic agents against lung cancer in Asian populations. His scientific area of interest is mainly focused on the study of the molecular and biological mechanisms of lung carcinogenesis and their clinical application. His research background also includes ILDs, their pathogenesis and potential treatments. In his lecture, Prof. Nakanishi shared insights into the Asian experience in the management of patients with coincident IPF and lung cancer, reviewed the possible risks in such populations and presented some recommendations from ongoing trials.

IPF is the most common ILD and is characterised by a radiological and/or pathological usual interstitial pneumonia (UIP) pattern leading to pulmonary fibrosis with reduced forced vital capacity and diffusing capacity of the lung for carbon monoxide [17]. Its prognosis is poor, with a median survival time of 3-5 years. The prevalence of co-existing lung cancer and IPF presents variably in different studies and ethnicities. From autopsy reports in Japan, the percentages of concurrent IPF and lung cancer fluctuate from $17 \%$ to $48 \%$ [18-21]. As far as western countries are concerned, lung cancer is found in $0-38 \%$ of patients with IPF [22], and the incidence of lung cancer in patients with interstitial lung diseases is seven to 14 times higher than in controls [23, 24].

The treatment of lung cancer combined with IPF or other ILD is challenging. There are no specific guidelines in western countries about optimal treatment for this subgroup of patients, for which the majority of new therapeutic targets pose a relatively high risk of acute exacerbation of ILD or drug-induced pneumonitis $[25,26]$.

During the perioperative period, life-threatening acute exacerbation of IPF is reported in up to $20 \%$ of patients [27]. The patients with resectable adenocarcinoma and UIP pattern on CT are mainly male, smokers and older, and the cancer is located in lower lobes with large size, low frequency of epidermal growth factor receptor (EGFR) mutation but high K-Ras mutation rates in histological biopsies [27]. Radiotherapy is generally contraindicated in patients with coinciding lung cancer and IPF [28].

Common genetic changes in the development of cancer and ILD have been reported, such as p53 mutations, loss of heterozygosity of the fragile histidine triad locus [29, 30], reduced expression of connexin-43 [31, 32] and abnormal tyrosine kinase activity [33, 34].

Moreover, smoking, exposure to toxic gas, infection and chronic damage tissue are the main risk factors for the epigenetic changes in both of these diseases [26], explaining why lung cancer and IPF show distinct pathophysiological similarities.

According to several studies, EGFR tyrosine kinase inhibitor (TKI)-induced ILD appears more common in Japanese than American or Korean patients. For instance, $4.5 \%$ of 3488 Japanese patients with non-small cell lung cancer (NSCLC) versus $0.3 \%$ of 23000 American patients developed ILD during therapy with erlotinib. The main risk factor was previous or concomitant ILD [35, 36]. As a result, EGFR-TKIs are not widely used in Japan for patients with NSCLC and IPF, despite encouraging results for progression-free survival (PFS) and overall survival (OS). There is also cautious use of immune checkpoint inhibitors because of the serious adverse events that may affect the lung, presenting as an ILD pattern. 
Therefore, it is necessary to design more studies of the management of patients with NSCLC in coexistence with IPF. The first ongoing randomised clinical trial in Japan aims to demonstrate that nintedanib combined with carboplatin plus nab-paclitaxel prolongs the interval to acute exacerbation of IPF compared with carboplatin plus nab-paclitaxel alone as well as to show that nintedanib could reduce the risk of chemotherapy-induced acute exacerbation of IPF [37]. Pirfenidone, orally administered to IPF patients with resectable NSCLC, significantly reduced perioperative, acute exacerbations of IPF in a multicentre, single-arm, phase II clinical trial [38] and phase III trials are now under consideration.

Lung cancer treatment has radically changed over the past decade. Novel targeted agents have emerged through the improved understanding of molecular pathways that lead to oncogenesis. More recent research has brought to light the various ways in which lung cancer cells evade the immune system, resulting in the development of agents targeting this activity. These novel therapies are checkpoint inhibitors targeting cytotoxic T-lymphocyte-associated antigen-4 and the programmed death (PD)-1 pathway, and have shown promising results, along with milder and manageable toxicities.

Solange Peters is in charge of medical oncology and thoracic malignancies at the Dept of Oncology of Lausanne University, Lausanne, Switzerland, and is currently ESMO President-Elect. Her scientific field of interest is the discovery of novel biomarkers in lung cancer, the combination of immunotherapies in NSCLC or small cell lung cancer treatment, and immunotherapy in stage III NSCLC with curative treatment options. In this session, she reviewed the biomarkers related to immunotherapy and the ongoing clinical trials for possible future routine application of these novel therapies.

Immunotherapy is nowadays an accepted therapy for advanced NSCLC in first and subsequent lines. Yet, it is important to better select patients eligible for immunotherapy, preventing potential harms in ineligible patients.

The PD-1 pathway is crucial in carcinogenesis as it inhibits T-cell activation. It is very important to clarify the definition of PD-1 ligand (PD-L1) positivity and the cut-off value in order to detect the patients that will benefit from anti-PD-1 immunotherapy. Moreover, there are five different drugs in this category so far (nivolumab, pembrolizumab, durvalumab, avelumab and atezolizumab) with different or no cut-off PD-L1 values. Other biomarkers, apart from PD-L1, should be developed to better predict response to immunotherapy. Tumour mutational burden (TMB) is being developed as a blood-based biomarker in advanced NSCLC [39] and could detect patients benefiting from immunotherapy. In 2017, the American Association for Cancer Research supported that TMB may not correlate with PD-L1 expression, instead showing an additive pattern. TMB by whole-exome sequencing (WES) is predictive of immunotherapy activity across diseases [40,41]. TMB by gene panel sequencing can replace WES and correlates with tumour-specific response rate [42]. An evolving cancer immunogram could facilitate decisions on certain treatment options in a more refined and personalised manner by visualising the state of cancer-immune system interactions [43].

Immunotherapy was first used as a second-line treatment. Pembrolizumab has been approved as first-line treatment for advanced NSCLC with PD-L1 $>50 \%$, with better outcome results than chemotherapy and significantly longer PFS [44]. Several studies have already been designed and completed in order to prove the efficacy of immunotherapy in first-line treatment with or without chemotherapy. For instance, carboplatin/taxane/pembrolizumab is superior to carboplatin/taxane for nonsquamous NSCLC, irrespective of PD-L1 expression, as far as PFS and OS is concerned [45]. Furthermore, the combination of platinum/pemetrexed with pembrolizumab was found to be more efficient in first-line therapy than chemotherapy alone [46]. The addition of atezolizumab to bevacizumab plus chemotherapy significantly improved PFS and OS among patients with metastatic nonsquamous NSCLC, regardless of PD-L1 expression and EGFR or anaplastic lymphoma kinase status [47]. In another study, the combination of ipilimumab/nivolumab was superior to platinum chemotherapy in NSCLC patients with TMB $>10 \mathrm{Mb}$, irrespective of PD-L1 [48].

There will be several changes in the approach to NSCLC treatment over the coming years. It is possible that targeted alterations will not play an important role in the selection of therapy, and we will focus only on PD-L1 and TMB. Patients with low TMB will receive chemo- and immunotherapy as a first-line treatment according to PD-L1 expression. Patients with high TMB and/or PD-L1 will mainly be eligible for sole immunotherapy.

Lung cancer remains a serious global health problem that affects millions of people each year and causes societies to pay a high toll not only in human lives but also in healthcare system resources. Over the past few years, new strategies aiming at prevention and early diagnosis have emerged. Furthermore, thanks to our improved understanding of the multiple mechanisms that lead to carcinogenesis and drug resistance, novel targeted therapies are used in everyday clinical practice that significantly improve survival, changing 
the landscape of the lung cancer treatment continuum. As demonstrated at the ERS International Congress 2018, healthcare professionals from all international respiratory societies share a vision for improving current lung cancer care, and aim to collaborate and push for prevention and early diagnosis as well as for high-quality, but also accessible, healthcare.

Conflict of interest: None declared.

\section{References}

1 World Health Organization. Cancer. www.who.int/news-room/fact-sheets/detail/cancer. Date last updated: September 12, 2018

2 American Cancer Society. Key statistics for lung cancer. www.cancer.org/cancer/non-small-cell-lung-cancer/about/ key-statistics.html. Date last updated: January 4, 2018.

3 International Early Lung Cancer Action Program Investigators. Survival of patients with stage I lung cancer detected on CT screening. N Engl J Med 2006; 355: 1763-1771.

4 National Lung Screening Trial Research Team. Reduced lung-cancer mortality with low-dose computed tomographic screening. N Engl J Med 2011; 365: 395-409.

5 Moyer VA, US Preventive Services Task Force. Screening for lung cancer: US Preventive Services Task Force recommendation statement. Ann Intern Med 2014; 160: 330-338.

6 National Comprehensive Cancer Network. Lung Screening. www.nccn.org/professionals/physician_gls/pdf/lung screening.pdf.

7 Kauczor HU, Bonomo L, Gaga M, et al. ESR/ERS white paper on lung cancer screening. Eur Radiol 2015; 25: 2519-2531.

8 American Thoracic Society, American Lung Association. Implementation Guide for Lung Cancer Screening. www. lung.org/assets/documents/lung-cancer/implementation-guide-for-lung.pdf.

9 Ali N, Lifford KJ, Carter B, et al. Barriers to uptake among high-risk individuals declining participation in lung cancer screening: a mixed methods analysis of the UK Lung Cancer Screening (UKLS) trial. BMJ Open 2015; 5: e008254.

10 Ten Haaf K, Jeon J, Tammemägi MC, et al. Risk prediction models for selection of lung cancer screening candidates: a retrospective validation study. PLoS Med 2017; 14: e1002277.

$11 \mathrm{Xu}$ DM, Gietema H, de Koning H, et al. Nodule management protocol of the NELSON randomized lung cancer screening trial. Lung Cancer 2006; 54: 177-184.

12 Field JK, Duffy SW, Baldwin DR, et al. UK Lung Cancer RCT Pilot Screening Trial: baseline findings from the screening arm provide evidence for the potential implementation of lung cancer screening. Thorax 2016; 71: $161-170$.

13 Pinsky PF, Gierada DS, Black W, et al. Performance of Lung-RADS in the National Lung Screening Trial: a retrospective assessment. Ann Intern Med 2015; 162: 485-491.

14 ten Haaf K, Tammemägi MC, Bondy SJ, et al. Performance and cost-effectiveness of computed tomography lung cancer screening scenarios in a population-based setting: a microsimulation modeling analysis in Ontario, Canada. PLoS Med 2017; 14: e1002225.

15 Archontogeorgis K, Steiropoulos P, Tzouvelekis A, et al. Lung cancer and interstitial lung diseases: a systematic review. Pulm Med 2012; 2012: 315918.

16 JafariNezhad A, YektaKooshali MH. Lung cancer in idiopathic pulmonary fibrosis: A systematic review and meta-analysis. PLoS One 2018; 13: e0202360.

17 Aburto M, Herráez I, Iturbe D, et al. Diagnosis of idiopathic pulmonary fibrosis: differential diagnosis. Med Sci (Basel) 2018; 6: E73.

18 Kawai T, Yakumaru K, Suzuki M, et al. Diffuse interstitial pulmonary fibrosis and lung cancer. Pathol Int 1987; 37: 11-19.

19 Matsuchita H, Tanaka S, Saiki Y, et al. Lung cancer associated with usual interstitial pneumonia. Pathol Int 1995; 45: 925-932.

20 Hironaka M, Fukayama M. Pulmonary fibrosis and lung carcinoma: a comparative study of metaplastic epithelia in honeycombed areas of usual interstitial pneumonia with or without lung carcinoma. Pathol Int 1999; 49: 1060-1066.

21 Qunn L, Takemura T, Ikushima S, et al. Hyperplastic epithelial foci in honeycomb lesions in idiopathic pulmonary fibrosis. Virchows Arch 2002; 441: 271-278.

22 Bouros D, Hatzakis $\mathrm{K}$, Labrakis $\mathrm{H}$, et al. Association of malignancy with diseases causing interstitial pulmonary changes. Chest 2002; 121: 1278-1289.

23 Hubbard R, Venn A, Lewis S, et al. Lung cancer and cryptogenic fibrosing alveolitis. A population-based cohort study. Am J Respir Crit Care Med 2000; 161: 5-8.

24 Le Jeune I, Gribbin J, West J, et al. The incidence of cancer in patients with idiopathic pulmonary fibrosis and sarcoidosis in the UK. Respir Med 2007; 101: 2534-2540.

25 Kanai O, Kim YH, Demura Y, et al. Efficacy and safety of nivolumab in non-small cell lung cancer with preexisting interstitial lung disease. Thorac Cancer 2018; 9: 847-855.

26 Bargagli E, Bonti V, Ferrari K, et al. Lung cancer in patients with severe idiopathic pulmonary fibrosis: critical aspects. In Vivo 2017; 31: 773-777.

27 Masai K, Tsuta K, Motoi N, et al. Clinicopathological, immunohistochemical, and genetic features of primary lung adenocarcinoma occurring in the setting of usual interstitial pneumonia pattern. J Thorac Oncol 2016; 11: 2141-2149.

28 Kolek V, Vašáková M, Šterclová M, et al. [Radiotherapy of Lung Tumours in Idiopathic Pulmonary Fibrosis]. Klin Onkol 2017; 30: 303-306.

29 Demopoulos K, Arvanitis DA, Vassilakis DA, et al. MYCL1, FHIT, SPARC, p16 ${ }^{\text {INK4 }}$ and TP53 genes associated to lung cancer in idiopathic pulmonary fibrosis. J Cell Mol Med 2002; 6: 215-222.

30 Uematsu K, Yoshimura A, Gemma A, et al. Aberrations in the fragile histidine triad (FHIT) gene in idiopathic pulmonary fibrosis. Cancer Res 2001; 61: 8527-8533. 
31 Trovato-Salinaro A, Trovato-Salinaro E, Failla M, et al. Altered intercellular communication in lung fibroblast cultures from patients with idiopathic pulmonary fibrosis. Respir Res 2006; 7: 122.

32 Yinn Y, Inase N. Connexin 43, E-cadherin, beta-catenin and ZO-1 expression, and aberrant methylation of the connexin 43 gene in NSCLC. Anticancer Res 2010; 30: 2271-2278.

33 Beyer C, Distler JH. Tyrosine kinase signaling in fibrotic disorders: translation of basic research to human disease. Biochim Biophys Acta 2013; 1832: 897-904.

34 Mitsudomi T. Advances in target therapy for lung cancer. Jpn J Clin Oncol 2010; 40: 101-106.

35 Nakagawa K, Kudoh S, Ohe Y, et al. Postmarketing surveillance study of erlotinib in Japanese patients with non-small-cell lung cancer (NSCLC): an interim analysis of 3488 patients (POLARSTAR). J Thorac Oncol 2012; 7 : 1296-1303.

36 Cohen MH, Johnson JR, Chen YF, et al. FDA drug approval summary: erlotinib (Tarceva) tablets. Oncologist 2005; 10: 461-466.

37 Otsubo K, Kishimoto J, Kenmotsu H, et al. Treatment rationale and design for J-SONIC: a randomized study of carboplatin plus nab-paclitaxel with or without nintedanib for advanced non-small-cell lung cancer with idiopathic pulmonary fibrosis. Clin Lung Cancer 2018; 19: e5-e9.

38 Iwata T, Yoshino I, Yoshida S, et al. A phase II trial evaluating the efficacy and safety of perioperative pirfenidone for prevention of acute exacerbation of idiopathic pulmonary fibrosis in lung cancer patients undergoing pulmonary resection: West Japan Oncology Group 6711 L (PEOPLE Study). Respir Res 2016; 17: 90.

39 Gandara DR, Paul SM, Kowanetz M, et al. Blood-based tumor mutational burden as a predictor of clinical benefit in non-small-cell lung cancer patients treated with atezolizumab. Nat Med 2018; 24: 1441-1448.

40 Carbone DP, Reck M, Paz-Ares L, et al. First-line nivolumab in stage IV or recurrent non-small-cell lung cancer. N Engl J Med 2017; 376: 2415-2426.

41 Hugo W, Shi H, Sun L, et al. Non-genomic and immune evolution of melanoma acquiring MAPKi resistance. Cell 2015; 162: 1271-1285.

42 Chalmers ZR, Connelly CF, Fabrizio D, et al. Analysis of 100,000 human cancer genomes reveals the landscape of tumor mutational burden. Genome Med 2017; 9: 34.

43 Blank CU, Haanen JB, Ribas A, et al. Cancer immunology. The "cancer immunogram". Science 2016; 352: 658-660.

44 Brahmer JR, Rodríguez-Abreu D, Robinson AG, et al. Health-related quality-of-life results for pembrolizumab versus chemotherapy in advanced, PD-L1-positive NSCLC (KEYNOTE-024): a multicentre, international, randomised, open-label phase 3 trial. Lancet Oncol 2017; 18: 1600-1609.

45 Paz Ares L, Luft A, Vicente D, et al. Pembrolizumab plus chemotherapy for squamous non-small-cell lung cancer. N Engl J Med 2018; 379: 2040-2051.

46 Ninomiya K, Hotta K. Pembrolizumab for the first-line treatment of non-small cell lung cancer. Expert Opin Biol Ther 2018; 18: 1015-1021.

47 Socinski MA, Jotte RM, Cappuzzo F, et al. Atezolizumab for first-line treatment of metastatic nonsquamous NSCLC. N Engl J Med 2018; 378: 2288-2301.

48 Hellmann MD, Ciuleanu TE, Pluzanski A, et al. Nivolumab plus ipilimumab in lung cancer with a high tumor mutational burden. N Engl J Med 2018; 378: 2093-2104. 